\title{
Quantitative Metrics for Assessing Positional and Orientational Order in Colloidal Crystals
}

\author{
Payam Bagheri, Ahmad M. Almudallal, Anand Yethiraj,* and Kristin M. Poduska* \\ Department of Physics, Memorial University of Newfoundland, St. John's, NL, Canada A1B 3X7
}

ABSTRACT: Although there are numerous self-assembly techniques to prepare colloidal crystals, there is great variability in the methods used to characterize order and disorder in these materials. We assess different kinds of structural order from more than 70 two-dimensional microscopy images of colloidal crystals produced by many common methods, including spin-coating, dip-coating, convective assembly, electrophoretic assembly, and sedimentation. Our suite of analysis methods includes
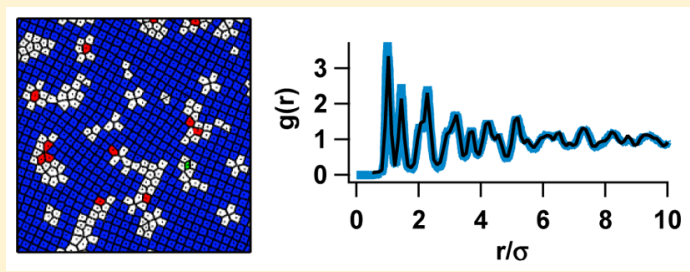
measures for both positional and orientational order. The benchmarks are two-dimensional lattices that we simulated with different degrees of controlled disorder. We find that translational measures are adequate for characterizing small deviations from perfect order, whereas orientational measures are more informative for polycrystalline and highly disordered crystals. Our analysis presents a unified strategy for comparing structural order among different colloidal crystals and establishes benchmarks for future studies.

\section{INTRODUCTION}

An important materials challenge in the last two decades has been to obtain perfect, defect-free colloidal crystals for use as photonic band gap materials. ${ }^{1-6}$ While this goal has thus far remained elusive, new applications have recently been proposed for colloidal films with lower degrees of order (such as substrates for surface-enhanced Raman spectroscopy ${ }^{7}$ ) or even amorphous structures (such as angle-independent structural color $^{8}$ ). This manuscript assesses, using different measures of positional and orientational order, the crystalline quality of $2 \mathrm{D}$ images of colloidal crystals, thus allowing systematic comparisons, with a single toolbox, of colloidal crystals reported in the literature.

Structural comparisons of colloidal crystals have often been based on visual inspection of microscopy images. ${ }^{4,9-11}$ Reciprocal space methods, either via Fourier transformation of optical images ${ }^{3,12}$ or via small-angle scattering methods, are useful in characterizing crystalline structures. ${ }^{13,14}$ However, spatial resolution is invaluable for studies of the kinetics of crystallization. Positional and orientational order, and correlations, are all convenient for characterizing time-independent and time-dependent processes on the same footing. ${ }^{15-19}$

A few recent studies have presented more quantitative assessments in real space. ${ }^{12,20}$ Portal-Marco et al. ${ }^{12}$ have compared colloidal structures composed of colloids with different size distributions by calculating positional and orientational correlation functions. Krejci et al. ${ }^{20}$ have assessed the order in a system of nanoparticles using Voronoi tessellation, a local bond-order parameter, an order parameter defined from the radial distribution function $g(r)$, and an anisotropy parameter that quantified orientational order. However, a detailed characterization of colloidal structures in terms of their local and overall translational and orientational order is still lacking.
In this work, we present a comprehensive comparison of structural order in colloidal crystals based on two classes of parameters: positional order and orientational order. We apply our metrics first to computer-generated structures ${ }^{21,22}$ to calibrate our assessments and then to a large collection of images of colloidal crystals. This includes images from the literature for samples prepared by a wide variety of techniques. ${ }^{1-5,9-11,23-40} \mathrm{We}$ also analyze large-area atomic force microscopy images of colloidal crystals that are new to this study.

\section{METHODS}

Structural Quantification. The positional coordinates of the particles in each image were determined using a well-established method introduced by Crocker and Grier. ${ }^{41}$ With these particle positions, we calculated the parameters summarized in Table 1.

Table 1. Summary of Quantification Parameters for Structural Order in Colloidal Crystals

local parameters

\begin{tabular}{ll}
\hline$\alpha$ & Lindemann parameter for positional disorder \\
$\Psi_{s}$ & local orientational bond order $(s=4$ or 6$)$ \\
$\left\langle\Psi_{s}\right\rangle$ & $\begin{array}{l}\text { average local orientation bond order }(s=4 \text { or } 6) \\
f_{4}, f_{6}, f_{d}\end{array}$ \\
$\sigma$ & $\begin{array}{l}\text { fraction of particles with } 4 \text {-fold, 6-fold, or disordered coordinations } \\
\text { most probable interparticle distance } \\
\text { long-range parameters }\end{array}$ \\
\hline$\xi_{s}$ & $\begin{array}{l}\text { positional correlation length within a single domain }(s=4 \text { or } 6) \\
\Delta_{s}\end{array}$ \\
$\begin{array}{l}\text { approximate domain size from diminishing orientational correlations } \\
(s=4 \text { or } 6)\end{array}$
\end{tabular}

Received: April 10, 2015

Revised: June 23, 2015

Published: July 3, 2015 
Table 2. Complete Set of Parameters Calculated for Images Used in This Study, Including Image Size $(x \times y)$, Effective Lindemann Parameter $(\alpha)$, Average Local Orientational Bond Order $\left(\left\langle\Psi_{s}\right\rangle\right)$, Symmetry Fraction $\left(f_{s}\right)$, Representative Domain Size $\left(\Delta_{4}\right)$, and Positional Correlation Length $\left(\xi_{s}\right)^{a}$

\begin{tabular}{|c|c|c|c|c|c|c|c|c|c|c|}
\hline image source & $x \times y$ & $\alpha$ & $\left\langle\Psi_{4}\right\rangle$ & $f_{4}$ & $\Delta_{4}$ & $\xi_{4}$ & $\left\langle\Psi_{6}\right\rangle$ & $f_{6}$ & $\Delta_{6}$ & $\xi_{6}$ \\
\hline \multicolumn{11}{|l|}{ Convective } \\
\hline Wong $(2003){ }^{2} 3 a$ & $94 \times 70$ & 0.038 & - & - & - & - & 0.96 & 1 & S & $*$ \\
\hline Wong $(2003),{ }^{2} 3 \mathrm{c}$ & $67 \times 49$ & 0.027 & - & - & - & - & 0.95 & 1 & $S$ & * \\
\hline Cong $(2003),{ }^{23} 4 \mathrm{c}$ & $29 \times 24$ & 0.050 & 0.89 & 0.39 & S & 12 & 0.92 & 0.47 & S & 5 \\
\hline $\mathrm{Gu}(2002),{ }^{25} 8$ & $20 \times 20$ & 0.020 & - & - & - & - & 0.98 & 1 & $S$ & 14 \\
\hline $\mathrm{Gu}(2002),{ }^{25} 8$ & $20 \times 20$ & 0.025 & - & - & - & - & 0.99 & 1 & S & 28 \\
\hline $\operatorname{Teh}(2005),{ }^{26} 4 a$ & $29 \times 22$ & 0.046 & - & - & - & - & 0.92 & 0.85 & S & 17 \\
\hline $\operatorname{Kuai}(2004),{ }^{10} 4 \mathrm{~b}$ & $76 \times 62$ & 0.027 & - & - & - & - & 0.98 & 1 & $S$ & 6 \\
\hline $\operatorname{Kuai}(2004),{ }^{10} 4 \mathrm{c}$ & $76 \times 62$ & 0.033 & - & - & - & - & 0.96 & 1 & $S$ & 7 \\
\hline $\operatorname{Kuai}(2004),{ }^{10} 4 \mathrm{~d}$ & $76 \times 62$ & 0.036 & - & - & - & - & 0.96 & 0.94 & S & 5 \\
\hline $\operatorname{Kim}(2005),{ }^{27} 1 \mathrm{a} \dagger$ & $98 \times 60$ & 0.0634 & - & - & - & - & 0.87 & 0.52 & $S$ & 2 \\
\hline $\operatorname{Kim}(2005),{ }^{27} 1 \mathrm{c} \dagger$ & $98 \times 65$ & 0.055 & - & - & - & - & 0.90 & 0.80 & $S$ & 9 \\
\hline $\operatorname{Kim}(2005),{ }^{27} 1 \mathrm{~d} \dagger$ & $98 \times 65$ & 0.027 & - & - & - & - & 0.96 & 0.97 & S & 6 \\
\hline $\mathrm{Ye}(2001){ }^{28} \mathrm{lb}$ & $31 \times 27$ & 0.028 & - & - & - & - & 0.97 & 1 & S & 14 \\
\hline \multicolumn{11}{|l|}{ Electrophoretic } \\
\hline Zhou(2013), ${ }^{29} 6 \mathrm{c}$ & $81 \times 56$ & 0.028 & 0.91 & 0.20 & $S$ & 7 & 0.96 & 0.71 & S & 5 \\
\hline Zhou $(2013),{ }^{29} 6 \mathrm{~d}$ & $36 \times 62$ & 0.019 & - & - & - & - & 0.98 & 1 & S & 10 \\
\hline Choi (2013), ${ }^{31} 3 \mathrm{~b} \dagger$ & $18 \times 18$ & 0.020 & - & - & - & - & 0.98 & 1 & S & 16 \\
\hline \multicolumn{11}{|l|}{ Electrospray } \\
\hline $\operatorname{Coll}(2013),{ }^{32} \mathrm{pcl}$ & $47 \times 35$ & 0.021 & - & - & - & - & 0.95 & 0.79 & 19 & 8 \\
\hline Coll(2013), ${ }^{32}$ pc2 & $170 \times 83$ & 0.040 & - & - & - & - & 0.90 & 0.53 & 25 & 3 \\
\hline Coll (2013),,$^{32} \mathrm{pc} 3$ & $55 \times 45$ & 0.026 & - & - & - & - & 0.98 & 0.97 & S & 7 \\
\hline Coll (2013), ${ }^{32}$ pc4 & $35 \times 29$ & 0.027 & - & - & - & - & 0.97 & 1 & $S$ & 29 \\
\hline \multicolumn{11}{|l|}{ Air-Liquid } \\
\hline Wang $(2011),{ }^{4} \mathrm{pcl}$ & $19 \times 13$ & 0.038 & - & - & - & - & 0.98 & 1 & S & $12^{*}$ \\
\hline Wang $(2011),{ }^{4} \mathrm{pc} 2$ & $24 \times 18$ & 0.034 & - & - & - & - & 0.97 & 1 & $S$ & $8^{*}$ \\
\hline Wang $(2011),{ }^{4} \mathrm{pc} 3$ & $21 \times 18$ & 0.030 & - & - & - & - & 0.98 & 1 & $S$ & $21^{*}$ \\
\hline Wang $(2011),{ }^{4} \mathrm{pc} 4$ & $21 \times 15$ & 0.043 & - & - & - & - & 0.97 & 1 & $S$ & $34^{*}$ \\
\hline Wang $(2011),{ }^{4} \mathrm{pc5}$ & $24 \times 17$ & 0.026 & - & - & - & - & 0.98 & 1 & $S$ & $6^{*}$ \\
\hline \multicolumn{11}{|l|}{ Confining Cell } \\
\hline $\operatorname{Park}(1998),{ }^{5} 2 \mathrm{a}$ & $77 \times 64$ & 0.023 & - & - & - & - & 0.97 & 0.96 & S & 12 \\
\hline Wang(2009), ${ }^{35} 2 \mathrm{~b}$ & $58 \times 61$ & 0.045 & - & - & - & - & 0.92 & 0.87 & $S$ & 4 \\
\hline Wang(2009),,$^{35} 2 \mathrm{~h}$ & $52 \times 32$ & 0.040 & - & - & - & - & 0.91 & 0.86 & S & 3 \\
\hline \multicolumn{11}{|l|}{ Dip-Coating } \\
\hline $\operatorname{Nagao}(2008),{ }^{36} 2 \mathrm{a} \dagger$ & $22 \times 24$ & 0.024 & - & - & - & - & 0.97 & 1 & S & 7 \\
\hline $\mathrm{Fu}(2008),{ }^{37} \mathrm{pc}$ & $23 \times 19$ & 0.051 & - & - & - & - & 0.93 & 1 & $S$ & $*$ \\
\hline \multicolumn{11}{|l|}{ Spin-Coating } \\
\hline $\operatorname{Mihi}(2006),{ }^{39} \mathrm{pcl}$ & $39 \times 29$ & 0.053 & 0.90 & 0.57 & 32 & 3 & 0.86 & 0.09 & 3.0 & $x$ \\
\hline $\operatorname{Mihi}(2006),{ }^{39} \mathrm{pc} 2$ & $39 \times 29$ & 0.054 & 0.89 & 0.52 & $S$ & 3 & 0.85 & 0.09 & 3.0 & $x$ \\
\hline $\operatorname{Mihi}(2006),{ }^{39} \mathrm{pc} 3$ & $39 \times 29$ & 0.048 & 0.87 & 0.35 & 17 & 5 & 0.90 & 0.30 & 16 & 3 \\
\hline $\operatorname{Mihi}(2006),{ }^{39} \mathrm{pc} 4$ & $37 \times 27$ & 0.059 & - & - & - & - & 0.90 & 0.85 & $S$ & 6 \\
\hline Jiang (2006), ${ }^{38} 2 \mathrm{~b} \dagger$ & $29 \times 24$ & 0.048 & - & - & - & - & 0.92 & 0.94 & $S$ & 4 \\
\hline Jiang $(2004),{ }^{3} 5 a$ & $87 \times 55$ & 0.039 & - & - & - & - & 0.94 & 0.93 & S & $13^{*}$ \\
\hline Cheng $(2014),{ }^{51} 5 b \dagger$ & $145 \times 106$ & 0.030 & - & - & - & - & 0.96 & 0.93 & 33 & 16 \\
\hline $10 E 40 \mu 3600 \dagger$ & $115 \times 115$ & 0.026 & - & - & - & - & 0.92 & 0.79 & 18 & 4 \\
\hline $10 E 50 \mu 4000 \dagger$ & $115 \times 115$ & 0.040 & - & - & - & - & 0.90 & 0.79 & 15 & 5 \\
\hline $10 E 50 \mu 4000 \dagger$ & $115 \times 115$ & 0.037 & - & - & - & - & 0.90 & 0.65 & 12 & 3 \\
\hline $10 E 50 \mu 4500 \dagger$ & $115 \times 115$ & 0.049 & - & - & - & - & 0.89 & 0.70 & 18 & 5 \\
\hline $10 E 50 \mu 4750 \dagger$ & $115 \times 115$ & 0.039 & - & - & - & - & 0.86 & 0.54 & 17 & 3 \\
\hline $15 E 40 \mu 2750$ & $115 \times 115$ & 0.081 & 0.82 & 0.06 & 5.0 & - & 0.85 & 0.52 & 18 & 5 \\
\hline $15 E 40 \mu 2750$ & $115 \times 115$ & 0.045 & 0.86 & 0.29 & 8.0 & 4 & 0.86 & 0.26 & 16 & 4 \\
\hline $15 E 40 \mu 3000$ & $115 \times 115$ & 0.046 & 0.87 & 0.47 & 14 & 5 & 0.84 & 0.12 & 7.0 & 4 \\
\hline $15 E 40 \mu 3250$ & $115 \times 115$ & 0.055 & 0.88 & 0.60 & 16 & 6 & - & - & - & - \\
\hline $15 E 40 \mu 4500$ & $115 \times 115$ & 0.038 & 0.87 & 0.25 & 8.0 & 4 & 0.87 & 0.35 & 20 & 3 \\
\hline $15 E 40 \mu 5000$ & $115 \times 115$ & 0.040 & 0.89 & 0.60 & 15 & 4 & - & - & - & - \\
\hline $15 E 40 \mu 5500$ & $115 \times 115$ & 0.038 & 0.90 & 0.60 & 15 & 4 & 0.82 & 0.05 & $x$ & $x$ \\
\hline $20 E 40 \mu 2000$ & $115 \times 115$ & 0.051 & 0.88 & 0.69 & S & 6 & - & - & - & - \\
\hline $20 E 40 \mu 2000$ & $115 \times 115$ & 0.034 & 0.89 & 0.65 & S & 6 & - & - & - & - \\
\hline $20 E 40 \mu 2000$ & $115 \times 115$ & 0.037 & 0.90 & 0.73 & $S$ & 7 & - & - & - & - \\
\hline
\end{tabular}


Table 2. continued

\begin{tabular}{|c|c|c|c|c|c|c|c|c|c|c|}
\hline image source & $x \times y$ & $\alpha$ & $\left\langle\Psi_{4}\right\rangle$ & $f_{4}$ & $\Delta_{4}$ & $\xi_{4}$ & $\left\langle\Psi_{6}\right\rangle$ & $f_{6}$ & $\Delta_{6}$ & $\xi_{6}$ \\
\hline \multicolumn{11}{|l|}{ Spin-Coating } \\
\hline $20 E 40 \mu 2000$ & $115 \times 115$ & 0.034 & 0.89 & 0.76 & $S$ & 7 & - & - & - & - \\
\hline $20 E 40 \mu 3000$ & $115 \times 115$ & 0.047 & 0.89 & 0.78 & $S$ & 9 & - & - & - & - \\
\hline $15 P 40 \mu 3000$ & $90 \times 90$ & 0.072 & 0.86 & 0.35 & 11 & 4 & 0.84 & 0.16 & 15 & 5 \\
\hline $15 P 40 \mu 5000$ & $115 \times 115$ & 0.082 & 0.87 & 0.66 & 11 & 6 & - & - & - & - \\
\hline $15 P 40 \mu 5000$ & $115 \times 115$ & 0.085 & 0.86 & 0.45 & 10 & 4 & - & - & - & - \\
\hline $20 P 100 \mu 2000$ & $115 \times 115$ & 0.054 & 0.84 & 0.08 & 5.0 & 3 & 0.87 & 0.50 & 20 & 5 \\
\hline $20 P 100 \mu 5000$ & $115 \times 115$ & 0.047 & - & - & - & - & 0.88 & 0.65 & 23 & 5 \\
\hline $20 P 100 \mu 5000$ & $115 \times 115$ & 0.058 & 0.88 & 0.66 & 17 & 5 & - & - & - & - \\
\hline $20 P 100 \mu 5000$ & $115 \times 115$ & 0.068 & 0.87 & 0.65 & 19 & 5 & - & - & - & - \\
\hline $20 P 10 \mu 7000$ & $115 \times 115$ & 0.054 & 0.84 & 0.17 & 9.0 & 3 & 0.83 & 0.26 & 18 & 3 \\
\hline $20 P 20 \mu 5000$ & $90 \times 90$ & 0.064 & 0.89 & 0.46 & $S$ & 5 & 0.88 & 0.21 & 15 & 4 \\
\hline $30 P 40 \mu 3000$ & $90 \times 90$ & 0.057 & 0.88 & 0.66 & $S$ & 5 & - & - & - & - \\
\hline $40 P 40 \mu 3000$ & $90 \times 90$ & 0.054 & 0.86 & 0.40 & 19 & 3 & 0.84 & 0.14 & 5.0 & 2 \\
\hline $40 P 40 \mu 3000$ & $90 \times 90$ & 0.058 & 0.87 & 0.59 & $S$ & 4 & 0.82 & 0.06 & 3.0 & $x$ \\
\hline \multicolumn{11}{|l|}{ Sedimentation } \\
\hline $\mathrm{Li}(2005),{ }^{40} 1 \mathrm{a}$ & $32 \times 27$ & 0.035 & - & - & - & - & 0.88 & 0.92 & S & $*$ \\
\hline $\mathrm{Li}(2005),{ }^{40} \mathrm{lb}$ & $32 \times 27$ & 0.039 & - & - & - & - & 0.91 & 0.98 & S & $9^{*}$ \\
\hline $\mathrm{Li}(2005),{ }^{40} 1 \mathrm{~d}$ & $32 \times 27$ & 0.027 & - & - & - & - & 0.89 & 0.96 & S & $11^{*}$ \\
\hline \multicolumn{11}{|l|}{ Random (CG3) } \\
\hline Ferry $(2011),{ }^{22} \mathrm{pcl}$ & $22 \times 22$ & 0.31 & 0.82 & 0.09 & 1.5 & $x$ & 0.80 & 0.11 & 2.0 & $x$ \\
\hline Ferry $(2011),{ }^{22}$ pc2 & $27 \times 27$ & 0.28 & 0.81 & 0.07 & 2.0 & $x$ & 0.79 & 0.13 & 3.5 & $\times$ \\
\hline Ferry $(2011),{ }^{22}$ pc3 & $30 \times 30$ & 0.23 & 0.82 & 0.07 & 2.0 & $x$ & 0.80 & 0.13 & 1.5 & $x$ \\
\hline
\end{tabular}
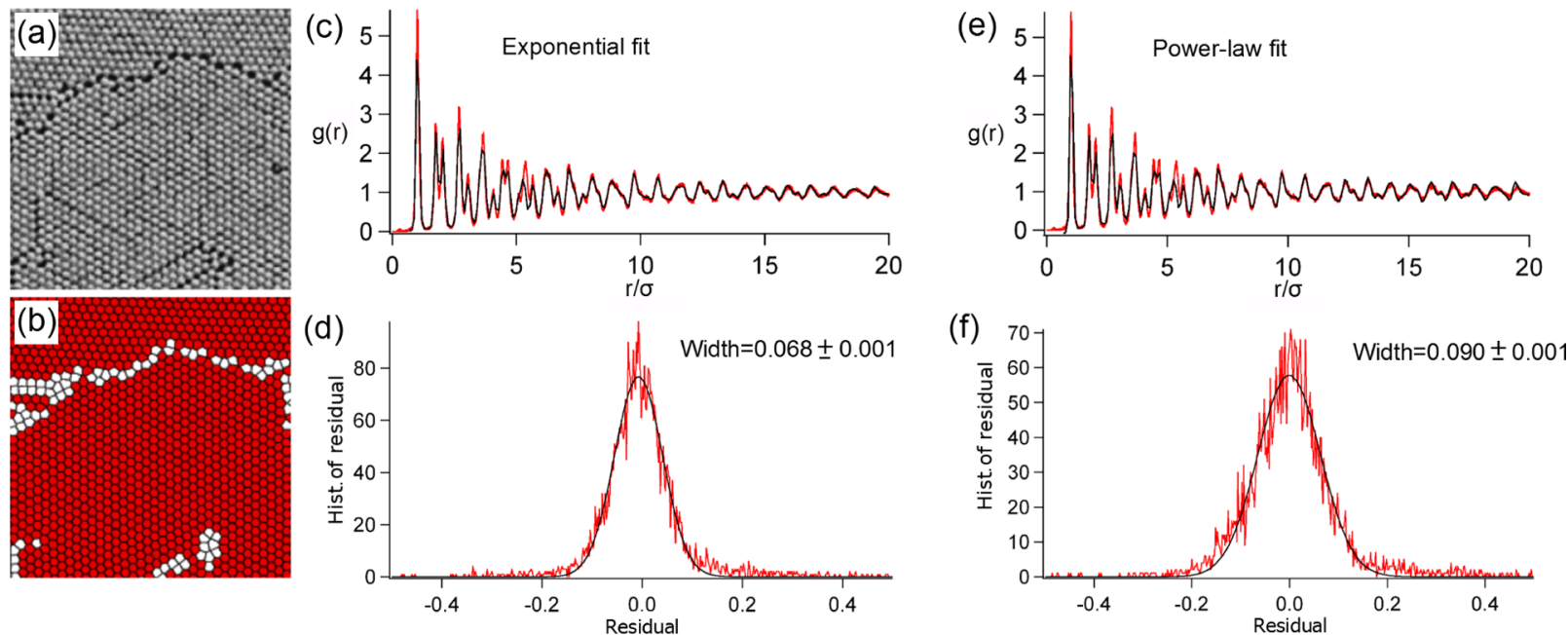

Figure 1. (a) A cropped SEM micrograph of a colloidal monolayer ${ }^{51}$ and (b) its local coordination color map (red = 6-fold coordination; white = disordered coordination). The $g(r)$ is compared with both an exponential $g_{\text {fit }}(c)$ and power law $g_{\text {fit }}$ (e) using histograms of the residuals for each fit (d, f). After fitting the histograms with a Gaussian form, the narrower width of the exponential fit leads us to favor it over the power law fit.

Local positional disorder is reported via the Lindemann parameter $\alpha$, defined as the standard deviation of the distribution of displacements divided by the ideal lattice spacing. ${ }^{42,43}$ For images from real colloidal crystals and the computer generated lattices labeled CG2, the distributions in nearest-neighbor distances were calculated over an entire image and fit with a Gaussian function to extract an effective $\alpha$. For computer generated lattices labeled CG1, $\alpha$ is the input parameter used to generate a Gaussian distribution of particle displacements in these images.

Local bond orientational order, defined as

$$
\Psi_{s}=\left|\frac{1}{N} \sum_{j=1}^{N} e^{i s \theta_{j}}\right|
$$

is assessed in the immediate neighborhood around a single lattice point. $^{44,45}$ Here, $N$ is the number of the nearest neighbors at that lattice point, and $\theta_{j}$ is the angle between a reference axis and the line connecting that lattice point to its $j$ th nearest neighbor. $\Psi_{s}$ is calculated relative to either perfect 4-fold $(s=4)$ or 6-fold $(s=6)$ symmetry, with a value between 0 and 1 , and 1 indicating perfect $s$-fold symmetry. We calculate $\Psi_{s}$ for both $s=4$ and 6 for each particle and choose $\Psi_{s}=0.7$ as the minimum threshold for $s$-fold order. The choice of 0.7 is arbitrary but reasonable. Lower $\Psi_{s}$ values are designated as disordered coordination. In very rare cases $(<0.1 \%)$, both $\Psi_{4}$ and $\Psi_{6}$ are larger than the threshold value, and the order cannot be determined. For easy visualization, we use color-coded images based on these assignments such that blue signifies $\Psi_{4}>0.7$, red indicates $\Psi_{6}>$ 0.7 , and white corresponds to disordered coordination. 
For each image used in this work, we report average bond orders $\left\langle\Psi_{s}\right\rangle$ to quantify the average values of $\Psi_{4}$ (for only particles designated as 4-fold sites) and $\Psi_{6}$ (for only particles designated as 6-fold sites). Furthermore, we assess the fraction $f_{s}(s=4,6$, or $d)$ of 6-fold, 4-fold, or disordered regions, respectively, within a single image.

Next, going beyond nearest neighbor order, we assessed the positional correlations across an entire image through the radial distribution function

$$
g(r)=\frac{1}{N}\left(\sum \frac{\mathrm{d} n}{\mathrm{~d} A}\right) /\left(\frac{N}{A}\right)
$$

Here, $\mathrm{d} n$ is the number of lattice points in a ring of inner radius $r$ and area $\mathrm{d} A, N$ is the total number of particles in the image, and $A$ is the total area of the image. We use the position $r=\sigma$ of the first maximum in $g(r)$ as a measure of the most probable interparticle spacing for a given image. By fitting this $g(r)$ with a complicated function, we extract a characteristic positional correlation length $\xi_{s}$ that we report relative to the most probable interparticle spacing $\sigma$.

It is well-known in the literature of $2 \mathrm{D}$ phase transitions that an exponential decay in a radial distribution function $g(r)$ indicates shortrange-order (such as for liquids), whereas a power law decay is consistent with quasi-long-range order (such as for $2 \mathrm{D}$ solids). ${ }^{15,46-49}$ An earlier report showed that the $g(r)$ for a two-dimensional solid can be fitted to distinguish between exponential and power law behaviors, such that ${ }^{50}$

$$
g_{\text {fit }}(r)=\left[\frac{A}{(2 \pi)^{(3 / 2)}} \frac{1}{\tilde{\sigma}} \sum_{i=1}^{n} \frac{g_{\text {id }}\left(x_{i}\right)}{D x_{i}} \exp \left(\frac{-\left(r-D x_{i}\right)^{2}}{2 \tilde{\sigma}^{2}}\right)-1\right] \times L(r)+1
$$

Here, $L(r)$ can be either $\exp (-r / \xi)$ for an exponential fit or $r^{-k}$ for a power law fit. We applied this fit to the radial distribution functions for all 69 colloidal crystal images listed in Table 2. An example is shown in Figure 1, and the details of our method follow below.

To use eq 3, we constrained several parameters during fitting. The peak widths are set by $\tilde{\sigma}=\sigma_{0} \sqrt{\ln \left(r / r_{0}\right)}$, where $r_{0}=0.3 D$, and $\sigma_{0}$ was limited to values between 0.03 and 0.08 . $D$ is the normalized lattice spacing, which we constrained between 0.8 and 1.1. The remaining parameters were unconstrained in our fits. $A$ is a scaling factor to adjust peak heights. $\xi$ is the positional correlation length (for the exponential fits), and $k$ is the exponent (for the power law fits).

The most computationally intensive part of the fit is the $g_{\text {id }}\left(x_{i}\right)$ term, which gives the number of particles at a distance $x_{i}$ from a point in a perfect lattice (either 6-fold or 4-fold, as appropriate). For example, $x_{1}$ would be the position of the first peak of the $g(r)$ in a perfect (hexagonal or square) lattice, and $g_{\text {id }}\left(x_{1}\right)$ would be the frequency of that peak position. We used up to $n=400$ points in the summation in the fit function over a range from $0 \leq r \leq r_{\max }$. The quality of the overall fit-especially in the first few $g(r)$ peaks -is influenced by the value of $r_{\max }$. For this reason, we investigated a statistical $\chi^{2}$ test, using the sum of squared errors between the true $g(r)$ and $g_{\text {fit }}(r)$, as a function of the fitting range separately for each image. In general, the statistical $\chi^{2}$ value grows as $r_{\max }$ increases until it reaches a plateau. Because of this, we chose the optimal $r_{\max }$ to be where the statistical $\chi^{2}$ plateaus. Beyond this range, the tail of the $g(r)$ function overwhelms the fit at the expense of the first peaks.

We applied the fit in eq 3 to all images listed in Table 2. This included images of true 2D colloidal monolayers as well as images that showed the top of multilayer structures (either quasi-2D or 3D). It was surprising that an exponential fit works well for all images. Figure 1 compares an exponential and a power law fit to the $g(r)$ for a representative image of a colloidal monolayer. In this case, we favor the exponential fit over the power law fit because of the narrower residual width. Thus, it is noteworthy that we see (liquidlike) exponential $g(r)$ decay trends even for structures that are ostensibly crystalline.

Finally, we also assessed orientational correlations among different domains within a single image. The orientational correlation function ${ }^{50}$

$$
g_{s}(r)=\left|\frac{1}{N_{B}} \sum_{l=1}^{N_{B}} \frac{1}{n_{l}} \sum_{k=1}^{n_{l}} e^{i s\left(\theta\left(r_{k}\right)-\theta\left(r_{l}\right)\right)}\right|
$$

is defined in terms of bonds $l$ between particles, and the angular orientations of these bonds relative to either 4 -fold $(s=8)$ or 6 -fold symmetry $(s=6)$. In the process of triangulation to identify bonds in a 4-fold structure, we can sometime encounter diagonal bonds. This can be accounted for by using $s=8$ for 4 -fold symmetric order parameters as was first suggested by Weiss and Grier. ${ }^{52}$ For the remaining parameters in eq 4, $\theta\left(r_{k}\right)$ and $\theta\left(r_{l}\right)$ are the angles between the bonds at $r_{k}$ and $r_{b}$, each with respect to the same reference axis. $n_{l}$ is the number of bonds at a distance $r$ from bond $l$, and $N_{B}$ is the total number of bonds in the structure. By assessing the orientational correlation function over an entire image, we use the first value of $r$ at which the correlations disappear $\left(g_{s}(r) \rightarrow 0\right)$ to indicate a representative size for a single domain; we denote this value as $\Delta_{s}$ and give its value relative to the most probable interparticle spacing $\sigma$.

Computer-Generated 2D Lattices. We used four different sets of $2 \mathrm{D}$ computer-generated lattices to compare with images of laboratory-produced colloidal structures. The first set of computergenerated (CG1) lattices has a predetermined degree of disorder, introduced by displacing each lattice point in a 2D crystal (with 4- or 6-fold symmetry) independently, such that there is a Gaussian distribution of displacements relative to their positions in the unperturbed crystal lattice.

The second set of computer-generated lattices (CG2) has disorder introduced in a more physically meaningful way by mimicking the effect of volume exclusion that occurs with nonzero particle sizes. We used 4-fold symmetric configurations obtained from Monte Carlo simulations of a $2 \mathrm{D}$ system of particles subject to a square-shoulder square-well potential ${ }^{21}$ calculated at different temperatures to generate different levels of disorder.

The third set of generated lattices (CG3) addresses the limiting case when there is no long-range positional or orientational order. These computer-generated random structures were obtained based on earlier published work. $^{22}$

Finally, multidomain crystals (CG4) were simulated by manually rotating a small number of perfectly ordered lattices (CG1 with no positional displacements) to create mosaics with arbitrarily rotated domains.

Two-Dimensional Images of Colloidal Crystals. We collected 40 scanning electron microscopy (SEM) and confocal fluorescence microscopy (CFM) images from the literature to span a wide range of common colloidal crystal assembly techniques: convective assembly, ${ }^{1,2,9-11,23-28}$ electrophoretic assembly, ${ }^{29-31}$ electrospraying, ${ }^{32}$ airliquid interface assembly, ${ }^{4,33,34}$ confinement, ${ }^{5,35}$ dip-coating, ${ }^{36,37}$ spincoating, ${ }^{3,38,39}$ and sedimentation. ${ }^{40}$

Additionally, 29 new atomic force microscopy (AFM) images were obtained from spin-coated colloidal crystals made by spin-coating $(2000-7000 \mathrm{rpm})$ a suspension of $0.46 \mu \mathrm{m}$ spherical silica particles $(10-40 \mathrm{vol} \%)$ in methyl ethyl ketone (MEK) or methyl propyl ketone (MPK) on glass substrates as described in earlier work by some of the same authors. ${ }^{17}$ Prior to AFM imaging (with Asylum Research MFP-3D in contact mode with Au-coated Si cantilevers (Mikromasch $\mathrm{CSC} 37 / \mathrm{Cr}-\mathrm{Au} / 50, k \sim 0.50 \mathrm{~N} / \mathrm{m}))$, the spin-coated colloidal crystals were coated with a thin $(\sim 50 \mathrm{~nm})$ spin-coated layer of poly(methyl methacrylate) (PMMA) to prevent displacement of the colloidal particles while scanning.

A comprehensive summary of the images, their sources, and our calculated parameters is given in Table 2. Images from the literature are named according to the paper from which they came, including the last name of the first author, its publication year, the reference citation, and its figure number. In cases where images were obtained through private communication, "pc" replaces a figure number. Our own images (new to this study) use a different naming convention. For example, "10MEK $40 \mu 3600$ " signifies 10 volume percent suspension of $0.460 \mu \mathrm{m}$ spherical silica particles in Methyl Ethyl Ketone using $40 \mu \mathrm{L}$ during spin-coating at $3600 \mathrm{rpm}$. A dagger $(\dagger)$ after an image name indicates that the colloidal crystal is monolayer; all other samples are 
multilayer. This is important to note because earlier studies have demonstrated that a high degree of order in a surface layer does not guarantee a high degree of order deeper inside a colloidal crystal. ${ }^{53,54}$ The full size of each image is listed as $x \times y$ in units of $\sigma$.

Each image has an effective Lindemann parameter $\alpha$. The remaining order parameter values (local orientational bond order $\left\langle\Psi_{s}\right\rangle$, symmetry fraction $f_{s}$, representative domain size $\Delta_{4}$, and positional correlation length $\xi_{s}$ ) were calculated for $s=4$ - and 6-fold regions separately in each image whenever possible. Where symmetry fractions or domain sizes were too small to calculate meaningful values, table entries show “-" or " $\times$ ". Single-domain images are indicated with $\Delta_{s}=\mathrm{S}$. Uncertainty magnitudes are as follows: $\delta \alpha$ is 2 in the last decimal digit; $\delta \Psi_{s}=0.04 ; \delta f_{s} / f_{s}=0.1$ for $f_{s}$ above 0.2 , and $0.1<\delta f_{s} / f_{s}<0.3$ for $f_{s}$ below 0.2. $\delta \Delta_{s} / \Delta_{s}=0.05 . \delta \xi_{s} / \xi_{s}=0.05$.

For some of the SEM and AFM images, $g(r)$ fits were poor until we modified the aspect ratio to compensate for image distortion (drift). The $\xi_{s}$ values extracted from rescaled images are noted with an asterisk $(*)$ in the table, based on these horizontal scaling factors: Wang $(2011) \mathrm{pc} 1=0.94, \operatorname{Wang}(2011) \mathrm{pc} 2=0.93, \operatorname{Wang}(2011) \mathrm{pc} 3=$ 0.93, Wang(2011)pc4 $=0.94$, Wang(2011)pc5 $=0.92$, Jiang(2004)5a $=0.90, \operatorname{Li}(2005) 1 \mathrm{~b}=0.88$, and $\operatorname{Li}(2005) 1 \mathrm{~d}=0.80$. There were four cases for which linear rescaling was not sufficient, and these have no positional correlation length value listed.

\section{RESULTS AND DISCUSSION}

We begin by examining order as obtained through local measures that depend only on the immediate (nearestneighbor) environment. We then compare this with parameters for order related to longer-range correlations. Finally, we apply both local and longer-range characterizations to images with multiple domains.

Orientational Order Correlates with Positional Order. We calibrated differences in local order for images of real colloidal crystals by comparing with data from computergenerated lattices (CG1, CG2, and CG3) with controlled levels of positional disorder. The first step was to assign each particle a coordination environment based on its local orientational order parameter $\Psi_{s}$.

Panels a-e in Figure 2 show representative examples of both computer-generated (CG1) and colloidal crystal lattices using color codes of blue, red, and white for 4-fold, 6-fold, and disordered coordinationsm, respectively. In computer-generated random lattices (CG3), most lattice points show disordered coordination, as expected. However, in real samples, the disordered coordinations tended to coincide with point defects and domain boundaries.

Our comparisons between real and computer-generated lattices show a useful correlation between average local orientational order and average local positional order. For each image, we calculated $\left\langle\Psi_{6}\right\rangle$ and $\left\langle\Psi_{4}\right\rangle$ for each symmetry separately. The measured $\alpha$ value and $\left\langle\Psi_{s}\right\rangle$ for each image are plotted in Figure 2f. Data for computer-generated lattices with a simple Gaussian distribution of displacements (CG1, solid curves) serve as an upper bound for $\left\langle\Psi_{s}\right\rangle$ at a given $\alpha$ value. This is true for both $s=4$ (blue) and $s=6$ (red). For images from real colloidal crystals, $\alpha$ falls in a narrow range above 0.03 but below 0.10 consistent with the Lindemann melting criterion. ${ }^{42}$ Conversely, highly disordered computer-generated structures (CG3) have liquidlike values $(\alpha \geq 0.2)$. We note that all of the images used in this study can be considered crystalline because they do not exhibit orientational order features (small dislocations) that are indicative of hexatic phases. ${ }^{21}$

We refer to all the order parameter measures discussed here as "local" because they are based on nearest-neighbor correlations. We find that the best indicator of local order for
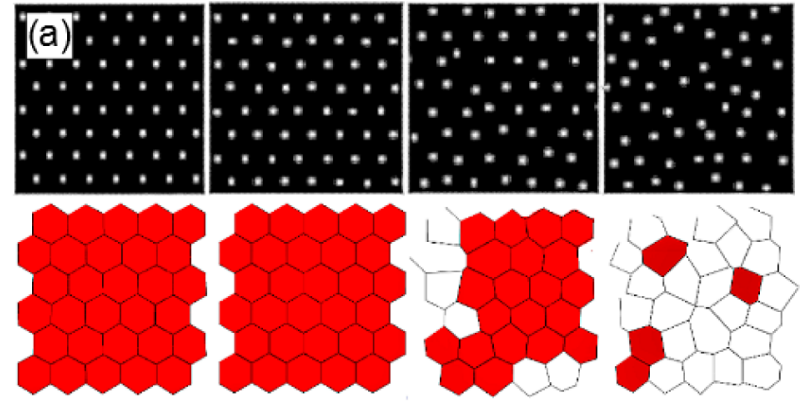

$\alpha=0.00$

0.02

0.08

0.15

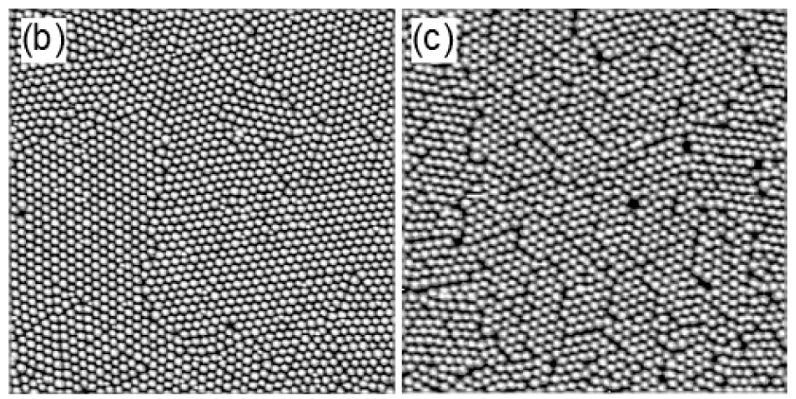

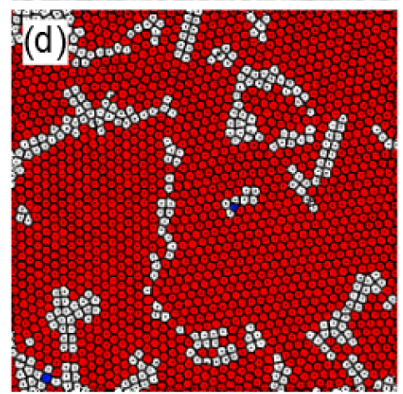

$a=0.026<\Psi_{6}>=0.92$

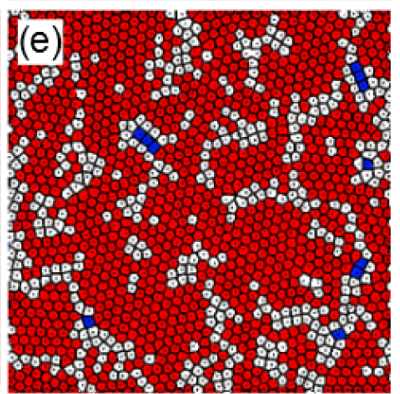

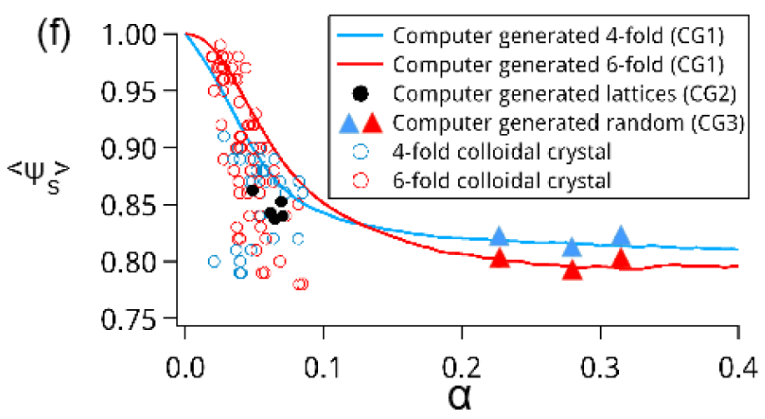

Figure 2. Local order parameters compared among computergenerated lattices and real colloidal crystals. (a) Representative computer-generated lattices (CG1) and (b-e) AFM images of spincoated colloidal monolayers. Red, blue, and white indicate 6-fold, 4fold, and disordered coordination, respectively. Effective $\alpha$ values are noted below each color-coded image. (f) Correlated average local bond order $\left\langle\Psi_{s}\right\rangle$ with values of $\alpha$. Trend lines for computer generated lattices (CG1), including those shown in (e), are shown as solid curves $(s=4(6)$ in blue (red)). Additional computer-generated images (CG2, CG3) are shown as solid black circles and solid triangles. The remaining data points, shown with open circles, are from images of real colloidal crystals.

real crystals is the parameter related to orientational order, $\left\langle\Psi_{s}\right\rangle$. In both simulated and experimentally obtained images, the average bond orientational order parameter $\left\langle\Psi_{s}\right\rangle$ is very high $\left(0.9<\left\langle\Psi_{s}\right\rangle<1\right)$ only when the positional disorder $\alpha$ is 

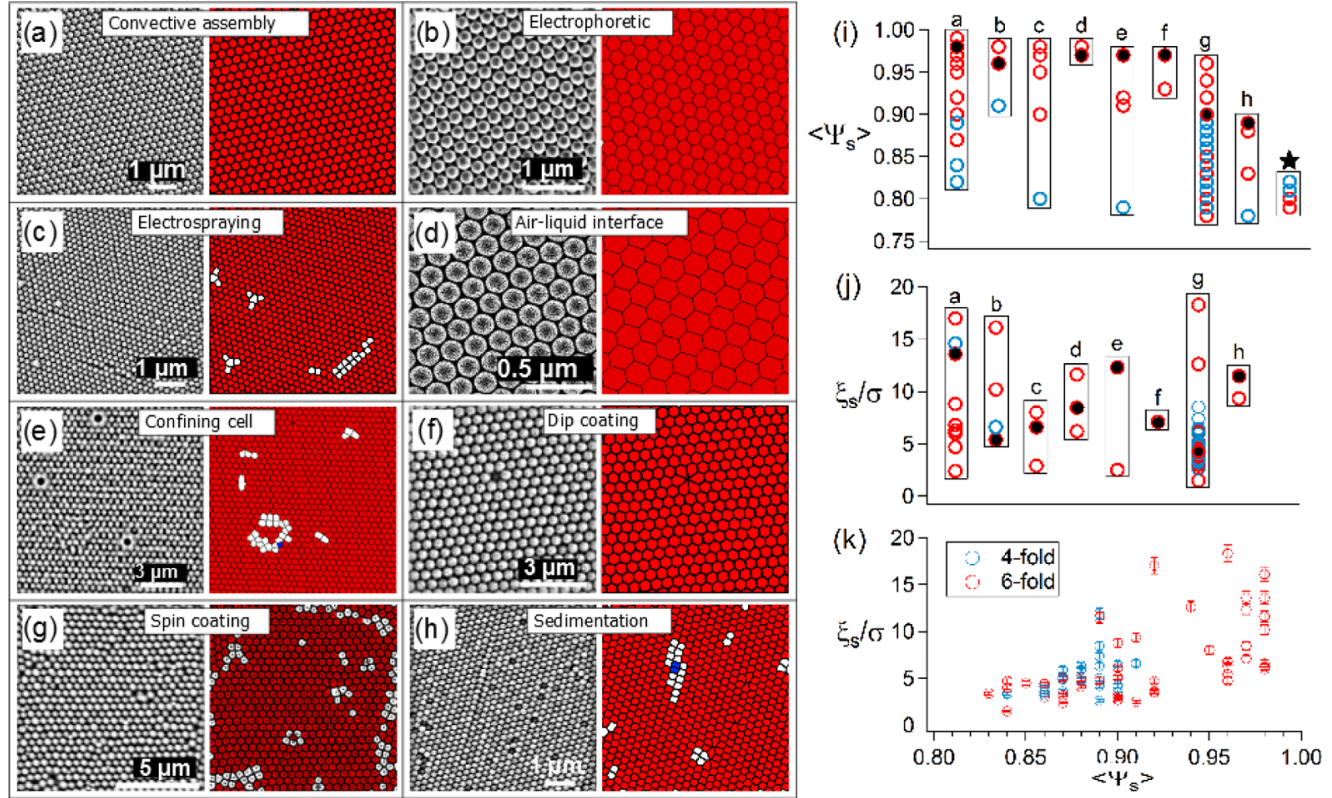

Figure 3. Representative images of colloidal crystals produced by (a) convective assembly, ${ }^{28}$ (b) electrophoretic assembly, ${ }^{29}$ (c) electrospraying, ${ }^{32}$ (d) air-liquid interface assembly, ${ }^{4}$ (e) confinement, ${ }^{5}$ (f) dip-coating, ${ }^{36}$ (g) spin-coating (new to this work), and (h) sedimentation. ${ }^{40}$ Comparisons of (i) average local orientational bond order $\left\langle\Psi_{6}\right\rangle$, and $(j)$ positional correlation length $\xi_{6}$, sorted according to production method. In (i) and (j), the black filled circles correspond to the data points for images $(\mathrm{a}-\mathrm{h})$. Data points marked with $(*)$ in (i) correspond to computer-generated random structures. ${ }^{22}$ Panel $(\mathrm{k})$ shows the correlation of $\xi_{s}$ with $\left\langle\Psi_{s}\right\rangle$, including data from colloidal crystals produced by all methods (whose details are listed in Table 2).

rather small $(0<\alpha<0.05)$. The shoulder in the simulated curves $\left(0.85 \approx\left\langle\Psi_{s}\right\rangle\right)$ highlights the region below which $\Psi_{s}$ is simply not very sensitive. This is especially evident in the data for the highly disordered computer-generated lattices (CG3), which are not equally random based on their different $\alpha$ values but have very similar $\left\langle\Psi_{s}\right\rangle$ values. Thus, in all subsequent discussions, we use $\left\langle\Psi_{s}\right\rangle$ as the primary local order parameter.

Intermediate-Range Correlations versus Local Orientational Order. Figure 3 examines correlations between local order (through $\left\langle\Psi_{s}\right\rangle$ ) and longer-range order (through the positional correlation length $\xi_{s}$ ). Because of the large number of images in this study, we grouped the data based on different colloidal crystal production techniques as shown in Figure 3: convective assembly, ${ }^{1,2,9-11,23-28}$ electrophoretic assembly, $^{29-31}$ electrospraying, ${ }^{32}$ air-liquid interface assembly, ${ }^{4,33,34}$ confinement, $^{5,35}$ dip-coating, ${ }^{36,37}$ spin-coating ${ }^{3,38,39}$ (the majority of which are original to this work), and sedimentation. ${ }^{40}$

On the basis of $\left\langle\Psi_{s}\right\rangle$ (Figure 3i) and $\xi_{s}$ data (Figure 3j), it is tempting to make quantitative comparisons of crystal quality among different production methods. For example, convective assembly appears to have the potential to produce crystals with the highest $\left\langle\Psi_{s}\right\rangle$. Convective assembly is also capable of producing colloidal crystals with relatively high $\xi_{s}$, with spincoating offering a competitive quality, although this technique in most cases seems to be poor in that regard. However, our sampling of data is implicitly biased because published images tend to be of the highest-quality areas rather than typical examples. This is why we made and measured a larger range of crystal images for one category of samples (spin-coating). It is worth noting that the best spin-coated samples are comparable to the best from convective assembly methods even though our data show that spin-coated crystals can be made with far lower degrees of positional correlation under some conditions.

Figure $3 \mathrm{k}$ shows the relation between positional correlation length $\xi_{s}$ and the average local (orientational) bond order $\left\langle\Psi_{s}\right\rangle$. $\xi_{s}$ is very small for $\left\langle\Psi_{s}\right\rangle<0.9$, which is another indication that crystals with $\Psi_{s} \leq 0.9$ do not correlate with good crystallinity. This is a reasonable result because good local orientational order is necessary for longer range positional correlations.

Distinguishing between Single Domains and Polycrystals. The metrics discussed thus far (i.e., $\left\langle\Psi_{s}\right\rangle, \alpha, \xi_{s}$ ) work well to characterize order within a single domain. This is sufficient for many images of colloidal crystals in the literature, due perhaps to the drive to make perfect photonic-grade crystals. However, more disordered crystals are also finding uses in optical applications. ${ }^{7,8}$ In an extreme, the average bond orientational order $\left\langle\Psi_{s}\right\rangle$ is not very informative for a polycrystalline sample containing grains with different symmetry (4-fold versus 6-fold). Instead, for polycrystalline colloidal materials (as are produced routinely by spincoating $\left.{ }^{3,16}\right)$, it is helpful to identify domain sizes. As we will show, these domain sizes are consistent with practical upper limits for the intradomain parameters $\left\langle\Psi_{s}\right\rangle$ and $\xi_{s}$.

We found a new and expedient way to assess representative domain sizes based on differences in orientational order. Figure 4 shows two representative orientational correlation functions $g_{s}(r)$ that have qualitatively different behaviors. When $g_{s}(r)$ is calculated over a single domain, a nonzero plateau appears for large $r$ values (Figure 4a,b). However, $g_{s}(r)$ decays to zero when the image contains multiple domains that disrupt the orientational correlation (Figure $4 \mathrm{c}, \mathrm{d}$ ). We define $\Delta_{s}$ as the normalized distance $(r / \sigma)$ at which the orientational correlations vanish. These $g_{s}(r)$ decays are replicated in simulated polycrystalline lattices (Figure $4 \mathrm{e}$ ).

A representative domain size derived from orientational correlations has two clear benefits for quantitative characterization of colloidal crystals. First, this parameter can distinguish a single domain with defects (Figure $4 \mathrm{a}$, with ill-defined $\Delta_{s}$ ) from a polycrystalline sample (Figure $4 \mathrm{c}$, with a finite $\Delta_{s}$ ). Second, the concept of a representative domain size provides a 

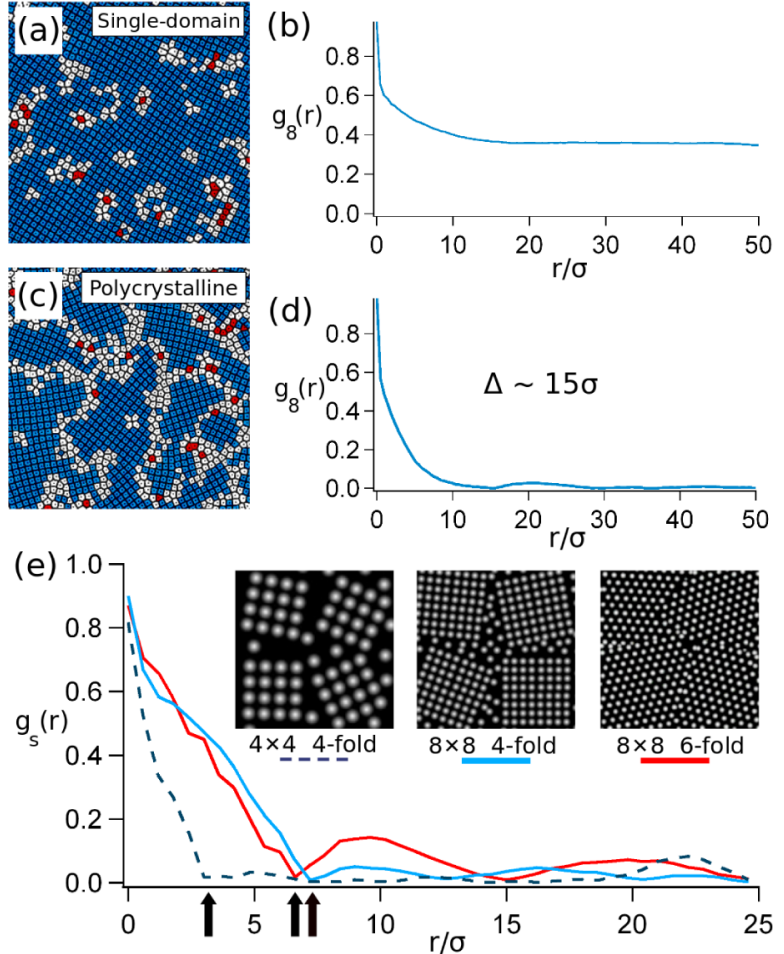

Figure 4. Representative orientational correlation functions $g_{s}(r)$. For a single domain (a), there is orientational correlation across the entire image, so $g_{s}(r)$ never reaches zero (b). Polycrystals (c) have orientational correlations that go to zero (d) at its representative domain size $\left(\Delta_{s}\right)$. (e) Examples of computer-simulated polycrystalline lattices and their corresponding $g_{s}(r)$ plots. Arrows show $\Delta_{s}$ values (where $g_{s}(r) \rightarrow 0$ ) for three simulated polycrystals.

reasonable upper bound for the useful range of intradomain characterization parameters such as $\left\langle\Psi_{s}\right\rangle$ and $\xi_{s}$. Figure 5 demonstrates that higher fractions of $s$-fold coordination $\left(f_{s}\right)$ correlate strongly with increases in both the average orientational bond order parameter $\left\langle\Psi_{s}\right\rangle$ and the representative orientational domain size $\Delta_{s}$. We note that, as demonstrated in Figure $5 \mathrm{~g}$, the orientational domain size is consistently larger than the translational correlation length for all colloidal crystals.

Finally, we address local order in polycrystals. The average bond order $\left\langle\Psi_{s}\right\rangle$ increases monotonically as the corresponding fraction $f_{s}$ increases (Figure 5e). When one symmetry dominates $(<0.1$ or $>0.9)$, small increases in $f_{s}$ lead to large increases in the average bond order. For crystals that contain a mixture of 4 - and 6-fold regions $(0.1<f<0.9)$, there is a more modest increase in the average bond order with increasing $f$. These results compare well with the trends observed for computer-generated structures (solid lines in Figure 5e). Our analyses show that, for a given $f_{s}$ value, real colloidal crystals have average bond order values that are consistently equal to or greater than those for computer-generated structures.

We emphasize that $\left\langle\Psi_{s}\right\rangle$ is calculated only within regions of specific symmetry (4- or 6-fold) and is not averaged over the entire image. Thus, the increase in average bond order with higher fractions is a true indication that the local order improves. It is not a trivial artifact related to averaging over ordered and amorphous regions. Figure 5f shows a strong correlation between increasing $f_{s}$ and increasing domain size $\Delta_{s}$.

\section{CONCLUSION}

Historically, a high degree of perfection was the goal for colloidal crystals for potential use in photonics. However, an increasing number of applications can take advantage of colloidal films with lower degrees of order (such as substrates for surface-enhanced Raman spectroscopy ${ }^{7}$ ) and even amorphous structures (such as angle-independent structural color ${ }^{8}$ ). In this context, we find that, for 2D characterizations of crystallinity, both orientational and translational measures are useful. For very good crystals, translational measures are often adequate because good translational order implies good orientational order. However, for crystals with higher levels of disorder that lead to polycrystallinity, the orientational measures, such as $\Delta_{s}$, are more sensitive indicators.

Although the positional correlation lengths are larger for samples with better orientational order, it is a noisy correlation. For example, excellent orientational order $\left(\left\langle\Psi_{s}\right\rangle=0.96\right)$ can be found in samples with either very short $(5 \sigma)$ or very large $(20 \sigma)$ $\xi_{s}$ values. Even in single-domain samples, $\xi_{s}$ is often limited. Considering the spread of $\xi_{s}$ and $\left\langle\Psi_{s}\right\rangle$ (Figure 3), the disappointing conclusion is that no single method can consistently produce only good crystals. Nevertheless, we found that all real crystals have crystallinity metrics ( $\alpha$ and $\xi_{s}$ ) that are far better than truly random computer-generated structures.

A useful finding is that the orientational correlation function $g_{s}(r)$ is a sensitive way to detect domain size in polycrystalline samples. This orientational domain size $\Delta_{s}$ shows a consistent increase as the symmetry fraction $f_{s}$ increases. It is also consistently larger than the positional correlation length. Furthermore, the average local orientational order $\left\langle\Psi_{s}\right\rangle$ in real samples is consistently better than values from simulated samples. This trend is nonlinear as a function of symmetry fraction, which shows once again that $\left\langle\Psi_{s}\right\rangle$ is a very sensitive order parameter for nearly single domain samples $\left(f_{s}=0\right.$ or 1$)$, whereas the symmetry fraction $f_{s}$ is more informative for mixed symmetry samples.

It is also interesting to note that we see good agreement with an exponential decay in the $g(r)$ functions in our images from crystalline samples. It is well-known in the literature of $2 \mathrm{D}$ phase transitions that an exponential decay suggests short-range order (as is typical for liquids) and that a power law decay implies quasi-long-range order (as is typical for 2D solids). ${ }^{15,46-49}$ Because the thickness of many samples in this study are not reported, it is unlikely that most published images correspond to true 2D crystals. Out-of-plane distortions are readily detected from AFM images in our spin-coated colloidal crystals, but such distortions are tricky to infer from SEM images. Furthermore, previous studies have demonstrated that there can be different degrees of order among surface and inner layers of multilayer colloidal crystals. ${ }^{53,54}$

From an overall perspective, the present study is valuable because it compares a large number of published images of colloidal crystals using the same assessment methods. Without this kind of a standardized approach, there are too many disparate methods of qualitative and semiquantitative characterizations that make it hard to assess advances in controlling order in these materials. The multiple characterizations of order that we use here are relatively straightforward to calculate. In addition, the analysis code we used is available to others upon request. Looking ahead, this set of analysis tools will enable 

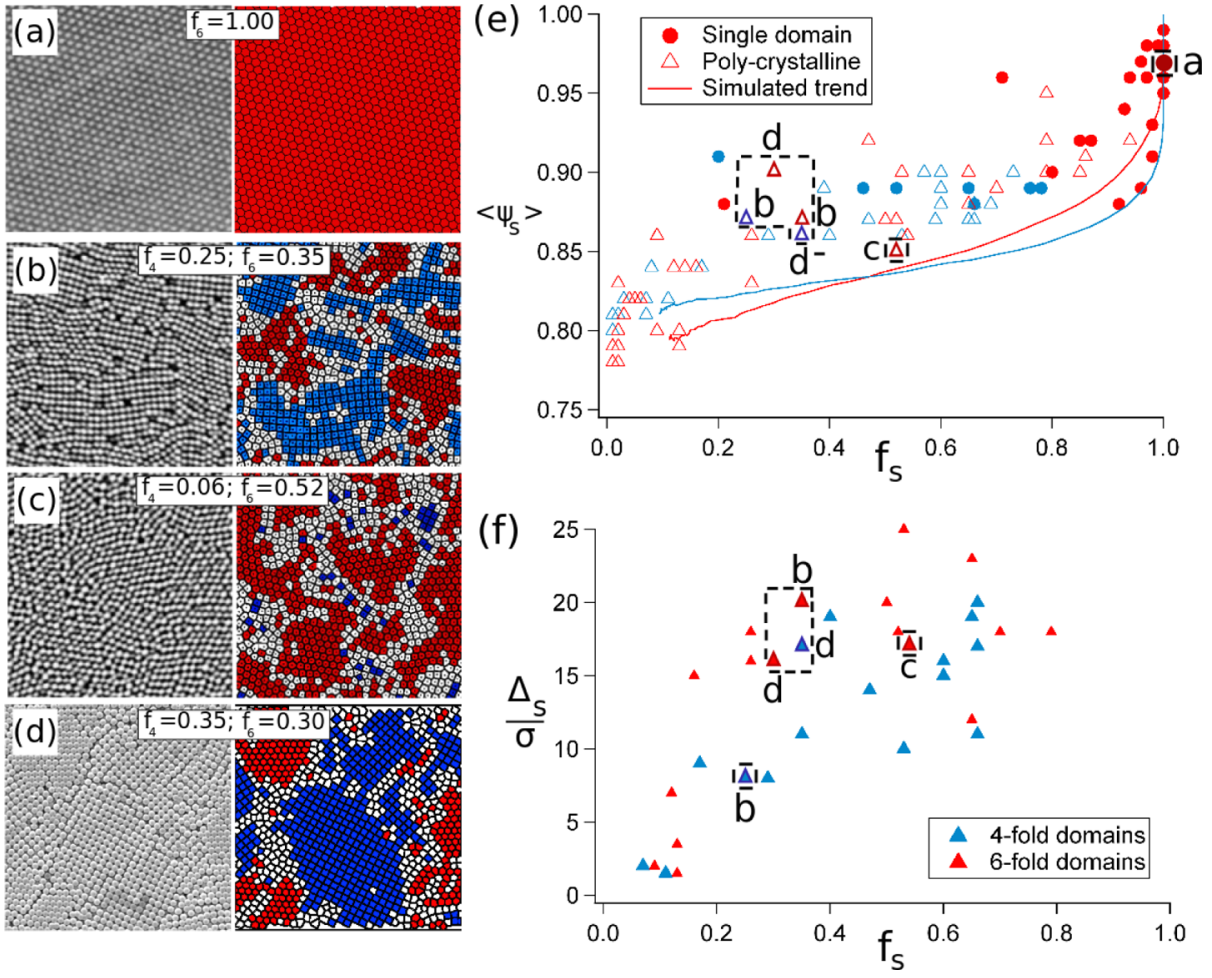

Figure 5. $(a-d)$ Four representative images with their associated local coordination color maps. In all panels, blue (red) means 4-fold (6-fold) local coordination. (e) Average local bond order $\left\langle\Psi_{s}\right\rangle$ improves with increasing symmetry fraction $f_{s}$ and is very sensitive when $f_{s} \geq 0.9$. Data points are for real colloidal crystals; trends for computer-generated structures are given by solid lines. In cases where a single domain spanned the entire image, the data points are shown as solid circles. The remaining (polycrystalline) structures are denoted with open triangles. (f) $\Delta_{s}$ vs $f_{s}$ shows correlation between these parameters. $\Delta_{s}$ values are reported only for polycrystalline samples. The data points corresponding to the images in (a-d) are marked by "a", "b", "c", and "d" in panels (e) and (f). Panel (a) is not represented by a point in panel (f) because the entire image is a single domain (the orientational correlation function never goes to zero but instead plateaus at $\left.g_{s}(r)=0.87\right)$.

quantitative comparisons between colloidal crystals from new experiments with images of older samples.

\section{AUTHOR INFORMATION}

\section{Corresponding Authors}

*E-mail: ayethiraj@mun.ca.

*E-mail: kris@mun.ca.

\section{Notes}

The authors declare no competing financial interest.

\section{ACKNOWLEDGMENTS}

We thank A. Coll, V. Ferry, Y. Fu, A. Mihi, and A.J. Wang for providing images that helped this study. We also thank I. SaikaVoivod for discussions on effective simulations of disordered colloidal crystals. A.Y. and K.M.P. thank the Natural Science and Engineering Resource Council (Canada) for funding.

\section{REFERENCES}

(1) Jiang, P.; Bertone, J. F.; Hwang, K. S.; Colvin, V. L. Single-Crystal Colloidal Multilayers of Controlled Thickness. Chem. Mater. 1999, 11, 2132-2140.

(2) Wong, S.; Kitaev, V.; Ozin, G. A. Colloidal Crystal Films: Advances in Universality and Perfection. J. Am. Chem. Soc. 2003, 125, 15589-15598.

(3) Jiang, P.; McFarland, M. J. Large-Scale Fabrication of Wafer-Size Colloidal Crystals, Macroporous Polymers and Nanocomposites by Spin-Coating. J. Am. Chem. Soc. 2004, 126, 13778-13786.

(4) Wang, A. J.; Chen, S.-L.; Dong, P.; Hu, C. T.; Sang, L. Fabrication of large-area and high-quality colloidal crystal films on nanocrystalline porous substrates by a room temperature floating self-assembly method. Thin Solid Films 2011, 519, 1798-1802.

(5) Park, S. H.; Qin, D.; Xia, Y. Crystallization of Mesoscale Particles over Large Areas. Adv. Mater. 1998, 10 (13), 1028-1032.

(6) Joannopoulos, J. D.; Johnson, S. G.; Winn, J. N.; Meade, R. D. Photonic Crystals, Molding the Flow of Light; Princeton University Press, 2008.

(7) Kuncicky, D. M.; Prevo, B. G.; Velev, O. D. Controlled assembly of SERS substrates templated by colloidal crystal films. J. Mater. Chem. 2006, 16, 1207-1211.

(8) Harun-Ur-Rashid, M.; Imran, A. B.; Seki, T.; Ishii, M.; Nakamura, H.; Takeoka, Y. Angle-Independent Structural Color in Colloidal Amorphous Arrays. ChemPhysChem 2010, 11, 579-583.

(9) Chung, Y. W.; Leu, I. C.; Lee, J. H.; Hon, M. H. Fabrication of high-quality colloidal crystals by a capillary-enhanced method. Appl. Phys. A: Mater. Sci. Process. 2004, 79, 2089-2092.

(10) Kuai, S.-L.; Hu, X.-F.; Hache, A.; Truong, V.-V. High-quality colloidal photonic crystals obtained by optimizing growth parameters in a vertical deposition technique. J. Cryst. Growth 2004, 267, 317324.

(11) Zhang, J.; Liu, H.; Wang, Z.; Ming, N. Assembly of high-quality colloidal crystals under negative pressure. J. Appl. Phys. 2008, 103, 013517.

(12) Portal-Marco, S.; Vallvé, M. A.; Arteaga, O.; Ignés-Mullol, J.; Corbella, C.; Bertran, E. Structure and physical properties of colloidal crystals made of silica particles. Colloids Surf., A 2012, 401, 38-47.

(13) Thijssen, J. H. J.; Petukhov, A. V.; 't Hart, D. C.; Imhof, A.; van der Werf, C. H. M.; Schropp, R. E. I.; van Blaaderen, A. Characterization of Photonic Colloidal Single Crystals by Microradian X-ray Diffraction. Adv. Mater. 2006, 18, 1662-1666. 
(14) Huber, P.; Bunk, O.; Pietsch, U.; Textor, M.; Geue, T. Grazing Incidence Small Angle X-ray Scattering on Colloidal Crystals. J. Phys. Chem. B 2010, 114, 12473-12479.

(15) Gasser, U. Crystallization in three- and two-dimensional colloidal suspensions. J. Phys.: Condens. Matter 2009, 21, 203101203124.

(16) Arcos, C.; Kumar, K.; González-Viñas, W.; Sirera, R.; Poduska, K. M.; Yethiraj, A. Orientationally correlated colloidal polycrystals without long-range positional order. Phys. Rev. E 2008, 77, 050402(R).

(17) Bartlett, A. P.; Pichumani, M.; Giuliani, M.; González-Viñas, W.; Yethiraj, A. Modified Spin-coating Technique to Achieve Directional Colloidal Crystallization. Langmuir 2012, 28, 3067-3070.

(18) Nöjd, S.; Mohanty, P. S.; Bagheri, P.; Yethiraj, A.; Schurtenberger, P. Electric field driven self-assembly of ionic microgels. Soft Matter 2013, 9, 9199-9207.

(19) Pichumani, M.; Bagheri, P.; González-Viñas, W.; Poduska, K. M.; Yethiraj, A. Dynamics, crystallization and structures in colloid spincoating. Soft Matter 2013, 9, 3220-3229.

(20) Krejci, A. J.; Thomas, C. G. W.; Dickerson, J. H. Statistical assessment of order within systems of nanoparticles: Determining the efficacy of patterned substrates to facilitate ordering within nanoparticle monolayers fabricated through electrophoretic deposition. Phys. Rev. E 2013, 87, 042307.

(21) Almudallal, A. M.; Buldyrev, S. V.; Saika-Voivod, I. Inverse melting in a two-dimensional off-lattice model. J. Chem. Phys. 2014, 140,144505 .

(22) Ferry, V. E.; Verschuuren, M. A.; van Lare, M. C.; Schropp, R. E. I.; Atwater, H. A.; Polman, A. Optimized Spatial Correlations for Broadband Light Trapping Nanopatterns in High Efficiency Ultrathin Film a-Si:H Solar Cells. Nano Lett. 2011, 11, 4239-4245.

(23) Cong, H.; Cao, W. Colloidal Crystallization Induced by Capillary Force. Langmuir 2003, 19, 8177-8181.

(24) Wang, L.; Zhao, X. S. Fabrication of Crack-Free Colloidal Crystals Using a Modified Vertical Deposition Method. J. Phys. Chem. C 2007, 111, 8538-8542.

(25) Gu, Z.-Z.; Fujishima, A.; Sato, O. Fabrication of High-Quality Opal Films with Controllable Thickness. Chem. Mater. 2002, 14, 760765.

(26) Teh, L.; Tan, N.; Wong, C.; Li, S. Growth imperfections in three-dimensional colloidal self-assembly. Appl. Phys. A: Mater. Sci. Process. 2005, 81, 1399-1404.

(27) Kim, M. H.; Im, S. H.; Park, O. O. Rapid Fabrication of Twoand Three-Dimensional Colloidal Crystal Films via Confined Convective Assembly. Adv. Funct. Mater. 2005, 15, 1329-1335.

(28) Ye, Y.-H.; LeBlanc, F.; Haché, A.; Truong, V.-V. Self-assembling three-dimensional colloidal photonic crystal structure with high crystalline quality. Appl. Phys. Lett. 2001, 78, 52-53.

(29) Zhou, C.; Han, J.; Guo, R. A facile strategy to colloidal crystals by drying condensed suspension droplets. J. Colloid Interface Sci. 2013, 397, 80-87.

(30) Dziomkina, N. V.; Hempenius, M. A.; Vancso, G. J. Towards true 3-dimensional BCC colloidal crystals with controlled lattice orientation. Polymer 2009, 50, 5713-5719.

(31) Choi, W. M. Simple and rapid fabrication of large-area 2D colloidal crystals for nanopatterning of conducting polymers. Microelectron. Eng. 2013, 110, 1-5.

(32) Coll, A.; Bermejo, S.; Hernández, D.; Castañer, L. Colloidal crystals by electrospraying polystyrene nanofluids. Nanoscale Res. Lett. 2013, 8, 26.

(33) Lu, Z.; Zhou, M. Fabrication of large scale two-dimensional colloidal crystal of polystyrene particles by an interfacial self-ordering process. J. Colloid Interface Sci. 2011, 361, 429-435.

(34) Ai-Jun, W.; Sheng-Li, C.; Peng, D.; Qian, Z.; Gui-Mei, Y.; GuCong, S. Self-Assembling of Colloidal Particles Dispersed in Mixture of Ethanol and Water at the Air-Liquid Interface of Colloidal Suspension at Room Temperature. Chin. Phys. Lett. 2009, 26 (8), 086104.

(35) Wang, X.; Husson, S. M.; Qian, X.; Wickramasinghe, S. R. Vertical cell assembly of colloidal crystal films with controllable thickness. Mater. Lett. 2009, 63, 1981-1983.
(36) Nagao, D.; Kameyama, R.; Matsumoto, H.; Kobayashi, Y.; Konno, M. Single- and multi-layered patterns of polystyrene and silica particles assembled with a simple dip-coating. Colloids Surf., A 2008, 317, 722-729.

(37) Fu, Y.; Jin, Z.; Liu, Z.; Liu, Y.; Li, W. Self-assembly of colloidal crystals from polystyrene emulsion at elevated temperature by dipdrawing method. Mater. Lett. 2008, 62, 4286-4289.

(38) Jiang, P.; Prasad, T.; McFarland, M. J.; Colvin, V. L. Twodimensional nonclose-packed colloidal crystals formed by spincoating. Appl. Phys. Lett. 2006, 89, 011908.

(39) Mihi, A.; Ocaña, M.; Míguez, H. Oriented Colloidal-Crystal Thin Films by Spin-Coating Microspheres Dispersed in Volatile Media. Adv. Mater. 2006, 18, 2244-2249.

(40) Li, Q.; Chen, Y.; Dong, P. Improvement of the quality of silica colloidal crystals by controlling drying. Mater. Lett. 2005, 59, 35213524.

(41) Crocker, J. C.; Grier, D. G. Methods of Digital Video Microscopy for Colloidal Studies. J. Colloid Interface Sci. 1996, 179, 298-310.

(42) Lindemann, F. The calculation of molecular vibration frequencies. Phys. Z. 1910, 11, 609-612.

(43) Stacey, F. D.; Irvine, R. D. Theory of melting: thermodynamic basis of Lindemann's law. Aust. J. Phys. 1977, 30, 631-640.

(44) Murray, C. A. In Bond-Orientational Order in Condensed Matter Systems; Strandburg, K. J., Ed.; Springer, 1991.

(45) Yethiraj, A.; Wouterse, A.; Groh, B.; van Blaaderen, A. Nature of an Electric-Field-Induced Colloidal Martensitic Transition. Phys. Rev. Lett. 2004, 92 (5), 058301.

(46) Murray, C. A.; Van Winkle, D. H. Experimental Observation of Two-Stage Melting in a Classical Two-Dimensional Screened Coulomb System. Phys. Rev. Lett. 1987, 58 (12), 1200-1203.

(47) Strandburg, K. J. Two-dimensional melting. Rev. Mod. Phys. 1988, 60 (1), 161-207.

(48) Quinn, R. A.; Cui, C.; Goree, J.; Pieper, J. B. Structural analysis of a Coulomb lattice in a dusty plasma. Phys. Rev. E: Stat. Phys., Plasmas, Fluids, Relat. Interdiscip. Top. 1996, 53 (3), R2049-R2052.

(49) Qi, W.; Gantapara, A. P.; Dijkstra, M. Two-stage melting induced by dislocations and grain boundaries in monolayers of hard spheres. Soft Matter 2014, 10, 5449-5457.

(50) Knapek, C. A. Phase Transitions in Two-Dimensional Complex Plasmas; Springer, 2011.

(51) Cheng, Y.; Jönsson, P. G.; Zhao, Z. Controllable fabrication of large-area 2D colloidal crystal masks with large size defect-free domains based on statistical experimental design. Appl. Surf. Sci. 2014, 313, 144-151.

(52) Weiss, J. A.; Oxtoby, D. W.; Grier, D. G.; Murray, C. A. Martensitic transition in a confined colloidal suspension. J. Chem. Phys. 1995, 103, 1180

(53) Yan, Q.; Zhou, Z.; Zhao, X. S. Inward-Growing Self-Assembly of Colloidal Crystal Films on Horizontal Substrates. Langmuir 2005, 21, 3158.

(54) Emoto, A.; Fukuda, T. Tailored assembly of colloidal particles: Alternative fabrication of photonic crystal or photonic glass. Appl. Phys. Lett. 2012, 100, 131901. 